\title{
PARYLENE ENCAPSULATED SUB-MICRON STRUCTURES FOR IMPLANTABLE BIOMEMS
}

\author{
K. Scholten and E. Meng ${ }^{*}$
}

Department of Biomedical Engineering, Viterbi School of Engineering, University of Southern California, USA

\begin{abstract}
We present a method for sub-micron machining of flexible, thin-film structures fully encapsulated in biocompatible polymer poly(chloro-p-xylylene) (Parylene C) that improves feature size and resolution by an order of magnitude compared to prior work. A low temperature electron beam lithography (EBL) process compatible with Parylene-coated silicon substrates was developed, and characterized using patterned $\mathrm{Ti}$ structures with critical dimensions down to $250 \mathrm{~nm}$, including conducting traces, serpentine resistors, and electrodes with a nano-patterned texture. Using this newly developed technique, the first flexible, free-film Parylene-Ti-Parylene devices with nanoscale components were fabricated and characterized. One application of these sub-micron structures encapsulated in Parylene is next generation minimally invasive implants. Thus, we also demonstrated a prototype high density Parylene-based microelectrode neural probe using our nanopatterning approach.
\end{abstract}

\section{INTRODUCTION}

Polymer MEMS on flexible substrates enable novel microdevices ideal for minimally invasive biomedical implants. The typical approach is to employ surface micromachined structures encapsulated in biocompatible polymers such as Parylene C, polyimide, or PDMS. The selection of polymeric substrates over conventional MEMS materials like silicon or glass conveys several advantages: low elastic moduli, high electrical resistivity, chemical resistivity, optical transparency, and mechanical flexibility. In particular, a major motivation for the use of polymers are their mechanical properties, especially for applications involving contact with soft tissue, such as retinal or neural microprobes, where rigid substrates can induce tissue damage due to mechanical mismatch, and subsequently trigger immune responses that impede performance and device lifetime. In recent years several successful examples of implantable polymer bioMEMS have been demonstrated, including implantable neural probes $[1,2]$, flow sensors, and pressure sensors [3, 4].

Despite the many advantages of polymeric micromachined devices, a major limitation remains - the poor feature resolution ( $>$ $5 \mu \mathrm{m})$ achievable with typical micromachining processes that are compatible with polymer substrates. While sub-micron fabrication is routinely employed on conventional MEMS materials, there has been limited work transitioning this capability to soft polymer substrates [5]. Nanoscale patterning has been applied to polymers to create flexible stamps and shadowmasks for fabrication on other substrates [6,7], but direct sub-micron fabrication on polymer surfaces, or for polymeric biomedical implants remains a technology gap [8]. Existing nanolithography protocols involve exposure of substrate materials to high temperatures and energies that exceed recommended operating conditions for most commonly used MEMS polymers. Methods such as nano-imprint lithography and self-assembly benefit from their highlyparallelized approach to cover large surface areas and have been used to pattern repeated arrays of simple structures on polymers [9, 10]. However, these techniques place restrictions on material choice and are pose difficulties in aligning multi-layer patterns, such as those required for many implantable microdevices.
As such, existing polymer MEMS cannot compete with silicon devices on feature density or feature size, placing limits on structures that can be easily produced and the resulting device capabilities. For example, polymer neural microprobes typically contain less than a dozen electrodes for stimulating or recording, whereas the state-of-the-art comprises silicon devices with more than a hundred electrodes per probe [11]. This limitation precludes polymer MEMS with the capabilities, complexity or minimal footprint achievable with conventional materials.

We developed a modified electron beam lithography (EBL) protocol that enables fabrication of biocompatible polymer MEMS with sub-micron feature resolution; our design consists of thin, free-film Parylene devices encapsulating titanium structures with feature size as small as $250 \mathrm{~nm}$. This resolution was achieved with minimal optimization and represents a starting point for achievable nanoscale resolution. We describe our fabrication process and present initial electrical and mechanical characterization, showing our devices as functional, flexible and robust. Finally, we present a demonstrative application of this technology: a prototype Parylenebased cortical neural microprobe, with a greater number and density of recording sites than any prior polymer probe. a)

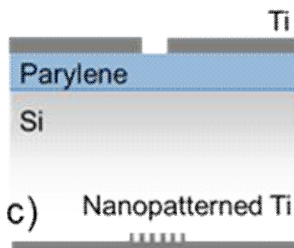

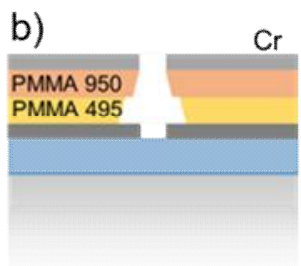

d) Parylene

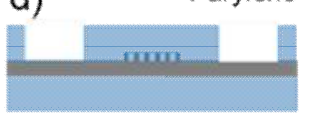

Figure 1: Process flow for sub-micron fabrication on Parylene C: (a) Ti contact pads and traces were fabricated on a vapor deposited Parylene film using conventional contact microlithography, (b) PMMA bilayer (PMMA 495, 950) was coated with $\mathrm{Cr}(15 \mathrm{~nm})$ then patterned with e-beam, (c) Ti features were deposited with lift-off, (d) features were encapsulated in Parylene contact pads were exposed with plasma etch, and device was released.

\section{METHODS}

\section{Fabrication}

The preparation of encapsulated structures (Figure 1) entailed a combination of conventional UV lithography, polymer micromachining, and a modified EBL method. First, silicon carrier wafers were coated in $10 \mu \mathrm{m}$ of Parylene $\mathrm{C}$ through chemical vapor deposition (CVD). Structures including alignment marks, contact pads and large electrical traces were patterned lithographically using a OAI hybralign series 200 mask aligner (OAI, San Joesa, CA), then metallized with titanium using e-beam evaporation and liftoff. A PMMA resist bilayer was prepared comprising $495 \mathrm{k} 2 \%$ solids in anisole (400 nm thick) and 950k 6\% 
solids in anisole (170 nm thick). Residual solvents were driven off with a $120^{\circ} \mathrm{C}$ hotplate bake for 20 minutes following deposition of each layer; the comparatively low baking temperature was necessary to avoid stress-induced Parylene cracking or polymer oxidation. A conductive chromium layer (10-15 nm) was deposited above the resist to prevent charge buildup during e-beam exposure, a consequence of the insulative properties of the Parylene layer. Representative images of the fabrication process development and results are shown in Figures 2-4.

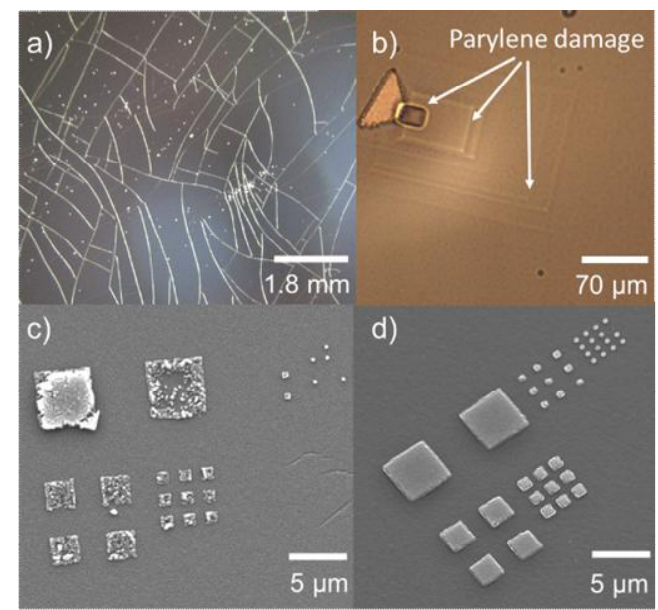

Figure 2: Sub-micron machining on Parylene $C$ under various conditions: (a) Parylene cracking induced by baking PMMA film at $170^{\circ} \mathrm{C}$, (b) damage to Parylene film due to uncalibrated e-beam exposure; and Ti features of decreasing size (5 - $0.25 \mu \mathrm{m})$ patterned on Parylene with (c) insufficient and (d) sufficient dose.

EB dose and voltage were varied $\left(150-900 \mu \mathrm{C} / \mathrm{cm}^{2} ; 10-20\right.$ $\mathrm{kV})$ to determine suitable parameters to achieve test patterns consisting of square arrays ( $5 \& 0.5 \mu \mathrm{m}$ sides). Then, squares of decreasing sizes $(5-0.05 \mu \mathrm{m})$ were patterned with an optimized dose $\left(420 \mu \mathrm{C} / \mathrm{cm}^{2} ; 20 \mathrm{kV}\right)$ to determine smallest resolvable features for the resist settings used (Fig. 2d). Test structures used in mechanical and electrical testing were patterned at the optimized dose, and consisted of straight and serpentine traces with widths and spacings of 5-0.25 $\mu \mathrm{m}$. Following exposure, the chromium layer was dissolved in CR-7 chromium etchant (Transene Company Inc., Danvers, MA), and the structures developed in 1:3 ratio of methyl isobutyl ketone to isopropanol (18-30s).

Dose and size calibration structures were successfully metallized with titanium and the resulting structures examined by scanning electron microscopy. Test structures were metallized with titanium $(150 \mathrm{~nm})$ and then encapsulated with a second CVD Parylene layer $(10 \mu \mathrm{m}) . \mathrm{O}_{2}$ deep reactive ion etching (DRIE) was used to expose the contact pads, and cut out test devices, which were then gently peeled off the silicon carrier to complete release of devices. No observable damage to the substrate or structures resulted from the peeling process.

\section{Mechanical and Electrical Testing}

DC resistance measurements were taken across all test traces using a basic 2-point probe on unstrained devices. For DC measurements during mechanical testing, electrical contacts were made using wire and conductive epoxy (silver epoxy, MG Chemicals, Surrey, CA). Devices were first manually bent and twisted while monitoring for changes in resistance or failures of the sub-micron trace. In a second set of experiments, devices were flexed using a moving stage and stepper motor, allowing resistance change to be monitored as a function of bend angle.
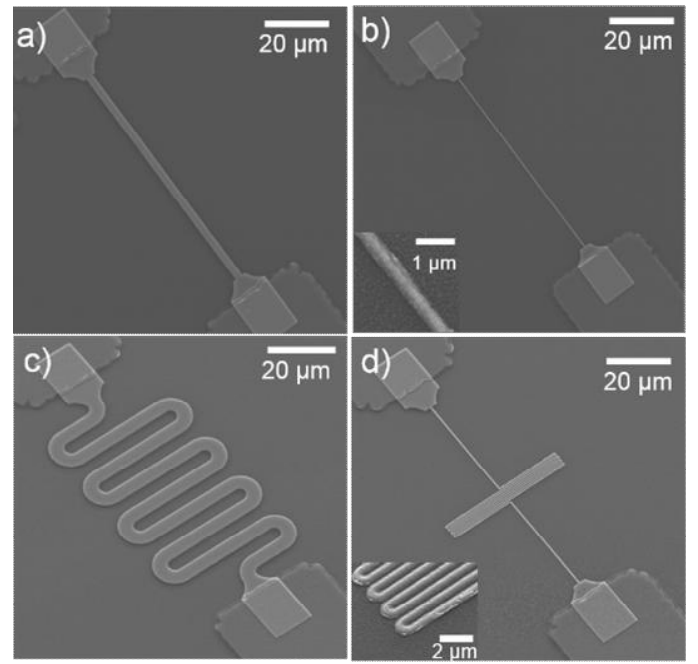

Figure 3: Representative SEM images of sub-micron Ti structures on Parylene: (a) straight $5 \mu \mathrm{m}$ trace, (b) straight $0.25 \mu \mathrm{m}$ trace with inset showing detailed view at higher magnification, (c) serpentine $5 \mu \mathrm{m}$ trace (5 $\mu \mathrm{m}$ spacing), and (d) serpentine $0.4 \mu \mathrm{m}$ trace (0.4 $4 \mathrm{~m}$ spacing).

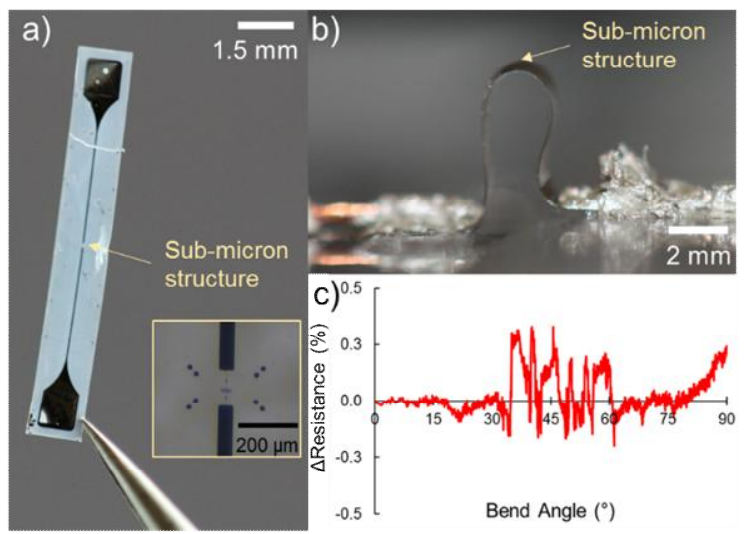

Figure 4: (a) Photograph of released and encapsulated test structure (400 $\mathrm{nm}$ serpentine Ti trace) with magnified inset. (b) Encapsulated device flexed while resistance is recorded. (c) Fractional change in resistance plotted against bend angle for representative test structure.

\section{Neural Probe Prototype}

A prototype cortical neural microprobe was fabricated using this method as a demonstrative example of a future application of sub-micron resolution Parylene devices. This microprobe consisted of a flexible Parylene shank $(2 \mathrm{~mm} \times 150 \mu \mathrm{m})$ supporting 32 electrodes, each patterned with arrays of nano-scale 'bump' structures $(400 \mu \mathrm{m})$ as a means to increase the effective surface area. Titanium electrode sites (15 $\mu$ m radius) were machined on a Parylene coated silicon wafer along with alignment marks using standard micromachining. The above EBL method was used to pattern the bump structures and connective electrical traces with width and spacing of $750 \mathrm{~nm}$. DRIE was used to cut-out the device shape, before removing it from the silicon carrier.

\section{RESULTS}

Careful optimization of the EBL process proved critical for 
working with the polymer substrate. The Parylene base layer exhibited cracking under thermal-induced stress and damage during high energy ebeam exposure. Subsequently, processing was tailored to avoid rapid heating or steps above $120^{\circ} \mathrm{C}$, and EBL was conducted at energies at or below $20 \mathrm{kV}$, with thick resist bilayers to assist in absorbing e-beam energies and protect the underlying Parylene. A metal surface layer proved necessary for high resolution EBL patterning, as without such a layer, surface charges collected and distorted the beam shape. Chromium was chosen due to its ease of removal, and low atomic mass which allowed prepatterned titanium structures to be visualized for alignment.

Exposure doses between 300 and $500 \mu \mathrm{C} / \mathrm{cm}^{2}$ at $20 \mathrm{kV}$ produced the smallest structures with most clearly defined features; structures patterned at lower doses or energies failed to develop fully while higher doses and energies produced distorted features or damaged Parylene film. Titanium structures with critical dimensions as small as $250 \mathrm{~nm}$ could be reliably machined, and exhibited strong adhesion to Parylene even under repeated deformation. Titanium was chosen owing to its demonstrated adhesion to Parylene and proven biocompatibility, though both platinum and gold were also tested, with the latter suffering from insufficient adhesion.

Features below $250 \mathrm{~nm}$ were not easily resolved, likely a limit of the achievable aspect ratio of the PMMA bilayer. Test devices with widths of 5-0.25 $\mu \mathrm{m}$, and spacing of 5-0.4 $\mu \mathrm{m}$ were successfully and repeatedly fabricated (Fig. 4) and proved flexible and robust. Serpentine traces with spacing of $0.25 \mu \mathrm{m}$ suffered from shorts and collapsed traces, perhaps a result of proximity exposure during patterning. Otherwise, under DC characterization, traces are Ohmic with no recorded shorts or faults even during repeated flexion and torsion (bend angle $>90^{\circ}$ ). Key values from mechanical and electrical testing are compiled in Table I, and Figure 5 displays trace resistance as a function of the length/width ratio, from which we calculate resistivity of the thin-film metal (3.5-6.4 $\mu \Omega \cdot \mathrm{m}$, compared to bulk values of $0.42 \mu \Omega \cdot \mathrm{m}$ ) and contact resistance (50-100 $\Omega$ ) at the interface between sub-micron structures and larger traces. Mean resistance was observed to vary by $<0.5 \%$ under flexion down to $500 \mu \mathrm{m}$ radius of curvature (Fig. $4 \mathrm{bc})$.

$\begin{array}{lcc} & \text { Straight } & \text { Serpentine } \\ \text { Dimension }(\mathrm{nm}) & 250 & 400 \\ \text { Resistivity }(\mu \Omega \mathrm{m}) & 6.35 & 3.54 \\ \text { Contact resistance }(\Omega) & 98 & 48 \\ \text { Torsion }\left({ }^{\circ}\right) & >90 & >90 \\ \text { Flexion }\left({ }^{\circ}\right) & >90 & >90\end{array}$

Table 1: Tabulated values are derived from electrical and mechanical characterization of straight and serpentine traces encapsulated in Parylene.

Figure 6 shows images of the prototype neural microprobe. The completed device had 32 recording sites on a $2 \mathrm{~mm}$ shank, more than double the electrode density of any prior polymer neural probe, and four times that of prior work done with Parylene C. This high density was achievable due to the small pitch and tight spacing of the connective electrical connections $(750 \mathrm{~nm})$, fabricated here through EBL. Traces extended the full length of the $2 \mathrm{~mm}$ shank without short or break, and exhibited the same flexibility as traces in test devices. Line resistance for the traces on the prototype was not measured, though can be extrapolated as 150 $\mathrm{k} \Omega$ from the regression plotted in Figure 5. Though larger than typical values of traces measuring several $\mu \mathrm{m}$ wide, at $1 \mathrm{kHz}$ (typical recording frequency for neural probe measurements) the contribution to impedance will be marginal.

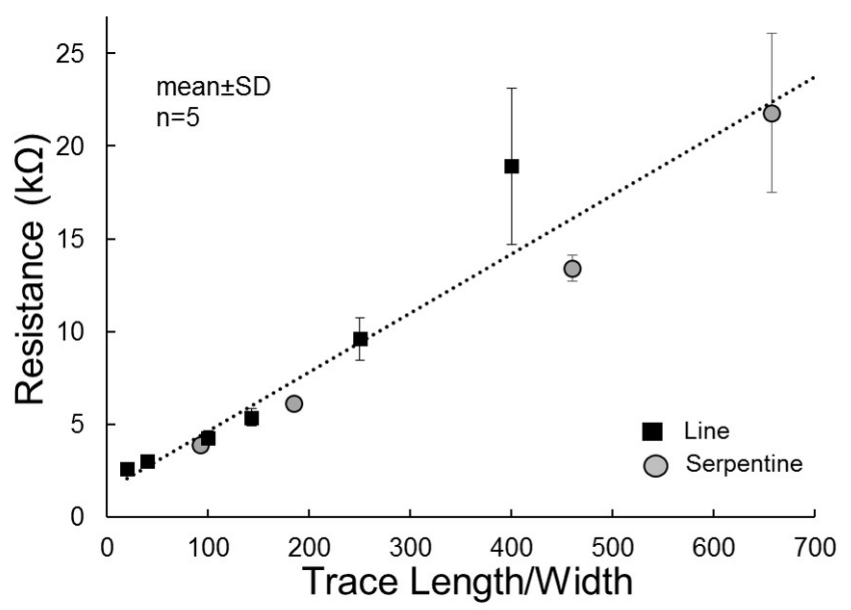

Figure 5 Plot of resistance vs. length-to-width ratio of patterned test structures: straight (square) and serpentine (circle) traces.

The nano-patterned structures, arrays of raised titanium bumps in the center of each electrode, served to increase effective surface area, and lower impedance. Each structure was rectangular, $150 \mathrm{~nm}$ tall and $400 \mathrm{~nm}$ across, and the arrays were calculated to increase effective surface area by $40 \%$.

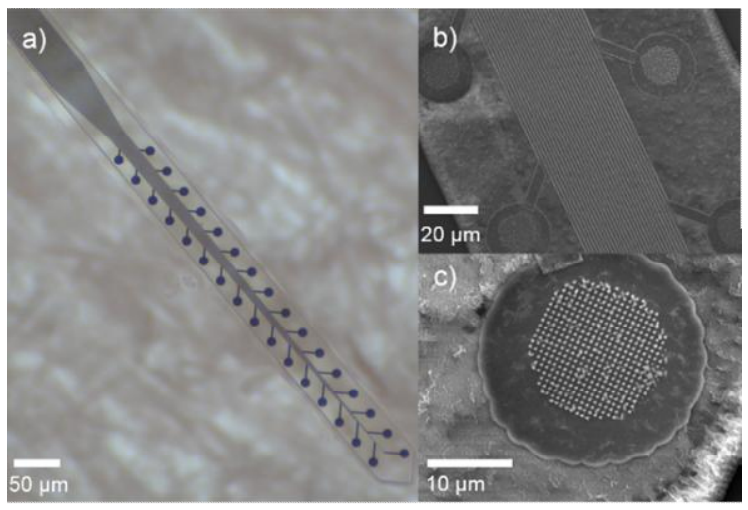

Figure 6 (a) Prototype Parylene-neural probe machined with submicron features including (b) densely packed conductive traces (750 nm linewidth and spacing) and (c) nano-patterned recording electrodes.

\section{DISCUSSION}

Through the use of the fabrication techniques described above, flexible Parylene-based devices were produced integrating electrical traces and structures with pitch and spacing of just a few hundred nanometers. Devices were flexible and robust, with insulated traces functioning despite severe and repeated flexion and torsion. These experiments bode well for the production of a new generation of polymer MEMS, with higher feature density and/or smaller footprints, and the highly biocompatible composition is encouraging for applying this technology to implants.

High resolution patterning on organic polymers is non-trivial; as seen here, issues of temperature, radiation intensity, and charge 
complicate existing methods developed for semiconductor applications. But these challenges are not unmanageable, and through careful preparation and calibration high resolution patterning, such as EBL, is achievable with high fidelity and good repeatability on such unconventional substrates. The resolutions achieved here were likely limited by the aspect ratio attainable with our choice of resist, and it is likely nanoscale fabrication is possible with only slight modification of this technique.

In on-going work, we are exploring other potential applications, including free-film devices with electrical components, such as resistors and capacitors, directed written into the film using dense serpentine traces and interdigitated comb designs respectively, or high density stimulating electrodes for neural or retinal devices, with large arrays of electrodes small enough to stimulate single sites of interest. We are cognizant of the scalability limitations of this work. Though EBL offers high resolution, the cost per device in time and money is too high for technologies intended for large scale distribution. This technique is useful for prototype devices intended for research, where the scale of production is small, or for prototyping novel devices. For other applications we are investigating pattern-transfer techniques compatible with Parylene that will enable batch-scale fabrication.

\section{CONCLUSIONS}

Despite material challenges electron beam lithography can be made compatible with organic polymers for MEMS processing. Through the use of such a technique, and the conformal nature of CVD Parylene, we have produced thin-film biocompatible devices with sub-micron features. This approach has applications for novel polymer implantable devices, demonstrated here through the production of a polymer neural microprobe with high electrode density. Sub-micron processing is a necessary advance for polymer MEMS, and future work will need to strive towards matching those resolutions achievable with silicon and other materials, to match their capabilities in mechanically pliant platforms.

\section{ACKNOWLEDGEMENTS}

This work was funded by a Postdoctoral Scholar Research Grant from the Office of the Provost at USC. The authors would like to thank Dr. Donghai Zhu of the Keck Photonics Laboratory for help with fabrication, and members of the Biomedical Microsystems Laboratory of USC for their assistance. Travel support was provided by OAI.

\section{REFERENCES}

[1]. B. Kim, J. Kuo, S. Hara, C. Lee, L. Yu, C. Gutierrez, T. Hoang, V. Pikov and E. Meng, "3D Parylene sheath neural probe for chronic recordings", Journal of neural engineering, 10, 4 (2013).

[2]. D. C. Rodger, A. J. Fong, W. Li, H. Ameri, A. K. Ahuja, C. Gutierrez, I. Lavrov, H. Zhong, P. R. Menon and E. Meng, "Flexible parylene-based multielectrode array technology for high-density neural stimulation and recording", Sensors and Actuators B: chemical, 132, 2 (2008).

[3]. K.-M. Lin, H. J. Sant, B. K. Ambati and B. K. Gale.

[4]. A. Sepúlveda, A. Pontes, J. Viana, R. G. de Villoria, F. Fachin, B. Wardle and L. A. Rocha, 2011.

[5]. K. Nagaraj, K. Sangeeth and G. Hegde, 2014.

[6]. C. P. Tan, B. R. Cipriany, D. M. Lin and H. G. Craighead, "Nanoscale resolution, multicomponent biomolecular arrays generated by aligned printing with parylene peel-off", Nano letters, 10, 2 (2010).
[7]. X.-M. Zhao, Y. Xia and G. M. Whitesides, "Soft lithographic methods for nano-fabrication", J. Mater. Chem., 7, 7 (1997).

[8]. K. Gonsalves, C. Halberstadt, C. T. Laurencin and L. Nair, Biomedical nanostructures, John Wiley \& Sons, 2007.

[9]. S. Aksu, M. Huang, A. Artar, A. A. Yanik, S. Selvarasah, M. R. Dokmeci and H. Altug, "Flexible plasmonics on unconventional and nonplanar substrates", Advanced Materials, 23, 38 (2011).

[10]. V. Auzelyte, B. Gallinet, V. Flauraud, C. Santschi, S. Dutta-Gupta, O. J. Martin and J. Brugger, "Large-Area Gold/Parylene Plasmonic Nanostructures Fabricated by Direct Nanocutting", Advanced Optical Materials, 1, 1 (2013).

[11]. K. Seidl, S. Herwik, T. Torfs, H. P. Neves, O. Paul and P. Ruther, "CMOS-based high-density silicon microprobe arrays for electronic depth control in intracortical neural recording", Microelectromechanical Systems, Journal of, 20, 6 (2011).

\section{CONTACT}

*E. Meng, tel: +1-213-821-3949; ellis.meng@usc.edu 